\title{
RESTAURANT EMPLOYEES' PERCEPTIONS OF BARRIERS TO THREE FOOD SAFETY PRACTICES
}

\author{
Amber D. Howells, MS, RD* \\ Research Assistant, Instructor \\ Hotel, Restaurant, Institution Management, and Dietetics \\ Kansas State University \\ 104 Justin Hall \\ Manhattan, KS 66506 \\ Phone: 785-410-4958 \\ Fax: 785-532-5522 \\ Email: geist78@ksu.edu \\ Kevin R. Roberts, PhD \\ Assistant Professor \\ Hospitality Management and Dietetics \\ Kansas State University \\ 104 Justin Hall \\ Manhattan, KS 66506 \\ Phone: 785-532-2399 \\ Fax: 785-532-5522 \\ Email: kevrob@ksu.edu

\section{Carol W. Shanklin, PhD, RD} \\ Associate Dean of the Graduate School and \\ Professor in Department of Hospitality Management and Dietetics \\ Kansas State University \\ 103 Fairchild Hall \\ Manhattan, KS 66506 \\ Phone: 785-532-7927 \\ Fax: 785-532-2983 \\ Email:shanklin@ksu.edu

\section{Valerie K. Pilling,PhD} \\ Psychology \\ Kansas State University \\ 492 Bluemont Hall \\ Manhattan, KS 66506 \\ Phone: 785-532-6850 \\ Fax: 785-532-5401 \\ Email: vpilling@ksu.edu
}




\author{
Laura A. Brannon, PhD \\ Associate Professor \\ Psychology \\ Kansas State University \\ 492 Bluemont Hall \\ Manhattan, KS 66506 \\ Phone: 785-532-0604 \\ Fax: 785-532-5401 \\ Email: lbrannon@ksu.edu \\ Betsy B. Barrett, PhD, RD \\ Associate Professor \\ Hospitality Management and Dietetics \\ Kansas State University \\ 104 Justin Hall \\ Manhattan, KS 66506 \\ Phone: 785-532-2208 \\ Fax: 785-532-5522 \\ Email: ebb@ksu.edu
}

*Denotes corresponding author

Keywords: food safety, perceived barriers, focus groups 


\title{
RESTAURANT EMPLOYEES' PERCEPTIONS OF BARRIERS TO THREE FOOD SAFETY PRACTICES
}

\author{
Funding Disclosure
}

This project was partially funded through a grant from the National Integrated Food Safety Initiative (Grant No. 2004-51110-02170) of the Cooperative State Research, Education, and Extension Service, U.S. Department of Agriculture. 


\title{
RESTAURANT EMPLOYEES' PERCEPTIONS OF BARRIERS TO THREE FOOD SAFETY PRACTICES
}

\begin{abstract}
Limited research has been conducted to assess employees’ perceptions of barriers to implementing food safety practices. Focus groups were conducted with two groups of restaurant employees to identify perceived barriers to implementing three food safety practices: handwashing, using thermometers, and cleaning work surfaces. Ten focus groups were conducted with 34 employees who did not receive training (Group A). Twenty focus groups were conducted with 125 employees after they had participated in a formal ServSafe ${ }^{\circledR}$ training program (Group B). The following barriers were identified in at least one focus group in both Group A and Group B for all three practices: time constraints, inconvenience, inadequate training, and inadequate resources. In Group A, additional barriers identified most often were a lack of space and other tasks competing with cleaning work surfaces; inconvenient location of sinks and dry skin from handwashing; and lack of working thermometers and thermometers in inconvenient locations. Additional barriers identified most often by Group B were no incentive to do it and the manager not monitoring if employees cleaned work surfaces; inconvenient location of sinks and dry skin from handwashing; and lack of working thermometers and manager not monitoring the use of thermometers. Results will be used to develop and implement interventions to overcome perceived barriers that training appears not to address. Knowledge of perceived barriers among employees can assist dietetic professionals in facilitating employees in overcoming these barriers and ultimately improve compliance with food safety practices.
\end{abstract}




\section{Restaurant Employees' Perceptions of Barriers to Three Food Safety Practices}

\section{Introduction}

\section{Background}

The restaurant industry plays a significant role in the economy of the United States. The estimated 935,000 restaurants in the United States generate $\$ 537$ billion in sales annually, representing $5 \%$ of the gross domestic product (1). Americans spend $47.9 \%$ of their annual food budget and consume $76 \%$ of their meals away from home (2). An estimated 70 billion meals and snack occasions will be eaten in American restaurants and other foodservice establishments in 2007 (2).

With the number of meals consumed in retail foodservice, assuring food safety should be very important to every restaurant manager and employee. Yet, a report issued by the Electronic Foodborne Outbreak Reporting System (FoodNet) indicated that 59\% of reported foodborne illness outbreaks were associated with restaurants in 2005 (3).

Given that the restaurant industry employs 12.8 million people (2), foodservice employees play an especially important role in preventing foodborne illness outbreaks (4). The top three factors resulting in foodborne illness outbreaks: poor personal hygiene, cross contamination, and time/temperature control are all directly related to food handler error (5). Limited research has been conducted to assess the impact of food safety training on employees' food safety practices (6-8). Previous research has found that food safety training increased knowledge regarding food safety issues (6). However, increased knowledge does not always translate into improved behaviors (7). Factors that have been found to negatively 
23 influence proper food handling practices included time pressures, resources, education and

24 training, and negative consequences (9-12). Research is needed to investigate barriers that

25 inhibit employees from practicing proper food safety. 31 thermometer. selected by contacting all local restaurants and asking the manager to permit researchers to

44 recruit their employees to participate in a food safety focus group. Restaurants contacted were

\section{Purposes}

The purpose of this study was to determine perceived barriers to performing three food safety practices: time/temperature control, personal hygiene, and cross contamination. Given that there are several food safety behaviors in these categories, the researchers selected three specific behaviors: handwashing, cleaning and sanitizing work surfaces, and using a

\begin{abstract}
Methodology
This exploratory study used two series of focus groups to assess restaurant employees' perceptions of barriers to implementing the three food safety practices at work. The methodology was reviewed and approved by Kansas State University’s institutional review board. Group A was composed of restaurant employees who had not completed a food safety class prior to the focus groups. Group B included employees who participated in focus group discussions immediately following a food safety class.
\end{abstract}

\section{Group A}

\section{Population and Sample}

Group A was composed of employees whose jobs involved food preparation in restaurants within the same city as the research institution. The convenience sample was to 
45 full-service and quick service restaurants, representing both independent and franchise

46 operations. For those who consented, sign-up sheets for multiple focus group sessions were

47 posted. Restaurant employees were offered \$20 in exchange for their participation. A total of 34

48 employees participated in 10 focus groups. Group A series of focus groups was the pilot phase

49 of this research. This series of focus groups were conducted to identify barriers that employees

50 perceived prevented them from implementing the three selected food safety practices. Results of

51 these focus groups were used to develop measurement items for a survey used in later phases of

52 data collection. Research protocol for using the Theory of Planned Behavior (13) states that it is

53 ideal to have approximately 25 people in the pilot phase of research (14). Thus, 34 participants

54 in this series of focus groups were adequate.

\section{Focus Group Interviews}

The purpose of Group A series of focus groups was to identify the most obvious barriers

57 to implementing food safety practices. Prior to the focus groups, participants were given a

58 questionnaire to complete, which contained the questions asked during the discussions. The

59 questionnaire gave employees the opportunity to think about their answers and was intended to

60 improve the quality of data obtained. Two to nine employees participated in each of the 10 focus

61 groups, which ranged in length from 40 to 60 minutes.

62 Participants signed informed consent forms and completed a demographic survey prior to

63 the discussions. Employees were asked to answer questions based on any job they had held

64 involving food production. Confidentiality of responses was ensured, and participants were

65 informed that only group data would be reported.

66 The interviewer familiarized the participants with the three food safety behaviors that

67 would be discussed. As each behavior was discussed, participants were asked what makes the 
68 behavior(s) difficult for them (or other employees) to engage in it. The interviewer allowed

69 sufficient time for the participants to contribute to the discussion while another researcher

70 documented the data by taking notes on a blank questionnaire with the focus group questions

71 listed. A response was documented if one person in a focus group stated the barrier. The same

72 researcher who documented the data also coded the focus group data. After the focus groups, the

73 researcher reviewed the data and developed categories of barriers for each behavior. Data were

74 managed by placing similar responses into categories for each behavior and writing those

75 responses on hard copy data sheets. The focus group responses were evaluated independently by

76 another researcher, who also grouped responses into categories for each behavior. Results were

77 compared and differences were discussed until 100\% consensus among the two researchers was

78 achieved.

\section{Group B}

$80 \quad$ Population and Sample

81 The population of Group B consisted of restaurant employees involved in food

82 production within a 300-mile radius of the research institution, including restaurants in Kansas,

83 Missouri, and Iowa. This radius was determined based on funding available to support this

84 project. For recruitment in Kansas and Iowa, a listing of operations licensed to sell food was

85 obtained from the state licensing agency. The telephone directory was used to recruit operations

86 in Missouri given that Missouri does not have a state-wide licensing system. A random sample

87 of 1,298 restaurants was contacted via telephone to request participation in the study. Different

88 sampling pools were used for Group A and Group B; no employees participated in both series of

89 focus groups. Researchers offered managers free food safety training for their employees as an 
90 incentive for participating in the project. If the manager agreed to participate, employees

91 involved in food preparation became part of the sample.

92 The recruitment period was from summer of 2005 to summer of 2006. A total of 20

93 restaurants completed this phase of the study. Managers facilitated employees' participation in

94 the training and focus groups. Employees received their hourly wage as compensation for their

95 participation. Restaurants that participated included full service, quick service, and catering

96 operations, representing both independent and franchise operations

\section{$97 \quad$ Training}

98 The food safety training was a four-hour session, using the ServSafe ${ }^{\circledR}$ Employee Guide

99 workbooks and videos. Trainers were certified ServSafe ${ }^{\circledR}$ instructors. The training sessions

100 included the importance of food safety, personal hygiene, receiving and storage, cooking and

101 holding food, and cleaning and sanitizing work surfaces. Multiple training sessions were

102 scheduled to accommodate the employees' schedules and ensure maximum participation. In

103 some cases, employees from multiple restaurants were trained together.

\section{Focus Group Interviews}

105 The purpose of Group B series of focus groups was to examine more subtle barriers that

106 could be identified by trained participants who had access to the more obvious barriers discussed

107 with the Group A participants. Participants completed a food safety knowledge assessment and

108 demographic survey following training. As stated in the instructions on the questionnaire, return

109 of the completed questionnaire served as informed consent. Employees then participated in

110 focus group discussions. A total of 125 employees participated in one of the 20 focus groups. A

111 larger sample size was utilized in this series of focus groups because we were no longer in the

112 pilot phase of the study. Therefore, all subjects that completed the training participated in Group 
113 B series of focus groups. Participants were given a focus group guide prior to the discussion that

114 contained the same questions asked during the focus groups for Group A. The guide also

115 included all responses from Group A and was designed to encourage participants to identify

116 additional barriers. Participants were able to freely discuss their responses and sufficient time

117 was allowed for participants to respond. The discussions lasted 15 to 30 minutes and were audio

118 recorded by using a digital recorder (Panasonic IC Recorder, Matsushita Electric Industrial Co.,

119 Ltd., Kadoma City, Osaka, Japan). Recordings were transcribed by a researcher, who

120 categorized data using the same coding scheme as for Group A. Group B series of focus groups

121 were audio recorded because multiple focus groups with more participants in each group were

122 involved in this phase of data collection. Therefore, there was more data to collect and manage at

123 one time. Audio-recording ensured more accurate and thorough coding of the data. Whereas,

124 Group A series of focus groups were not audio recorded because there were fewer people

125 overall, fewer focus groups in this series, and fewer participants in each group discussion. Group

126 A series of focus groups was a preliminary, smaller-scale data collection. Although the data was

127 coded thoroughly and accurately, it was not as much data to manage at one time. SPSS for

128 Windows (version 12.0, 2004, SPSS Inc., Chicago, IL) was used to organize the categories and 129 responses of Group A and Group B series of focus groups.

\section{Results and Discussion}

Group A listed a total of 43 barriers for the three behaviors. Results of Group A focus

132 groups were used to develop an instrument to evaluate perceived barriers to implementing food

133 safety practices that would be used later with a larger sample. Participants listed 15 barriers for

134 cleaning and sanitizing work surfaces, and 14 barriers each for handwashing and using a

135 thermometer (Table 1). 

using a thermometer, and 12 for handwashing. Table 1 summarizes the barriers discussed by

138 Group A and B for all three practices.

The barriers to cleaning and sanitizing work surfaces discussed most often by Group A

140 focus groups were time constraints (mentioned in 10 of 10 focus groups), inadequate

141 training/knowledge (8 of 10), and management and employees don’t care (8 of 10). Time

142 constraints (18 of 20), no incentive or desire to perform the practice (15 of 20), and management

143 and employees don't care (13 of 20) were the barriers identified most frequently for cleaning and

144 sanitizing work surfaces by Group B.

145 The three barriers mentioned most often by Group A for handwashing were time

146 constraints (10 of 10), resources in inconvenient locations (8 of 10), and dry skin (7 of 10).

147 Group B identified the following barriers for proper handwashing most often: time constraints 148 (14 of 20), inadequate resources (13 of 20), and dry skin (11 of 20).

149 The four barriers mentioned most often by Group A for using thermometers were time 150 constraints (9 of 10), lack of working thermometers (9 of 10), not knowing temperatures (7 of 151 10), and not knowing how to take temperatures (7 of 10). The four barriers identified by Group 152 B most often included inadequate training (13 of 20), lack of working thermometers (11 of 20), 153 not enough thermometers (10 of 20), and time constraints (7 of 20).

\section{Conclusions and Implications}

156 inadequate training, time constraints, inadequate resources, and inconvenience to perform the 157 practices. Given that the current study elicited barriers from both untrained and trained

158 employees, the authors are confident that the present list of barriers is fairly comprehensive and 
159 should be addressed in training programs. Even though this research was conducted with

160 restaurant operations, the results can be used to identify barriers in non-commercial foodservice

161 operations. It is important for registered dietitians (RDs), dietetic technicians registered (DTRs),

162 and foodservice managers to develop programs in their facilities that address these barriers if

163 food safety practices are to improve. Employees would be more likely to improve their food

164 safety behaviors if they perceive fewer barriers to properly performing them. For example, if

165 employees believe that they have enough time to properly wash their hands, they are more likely

166 to wash them.

167 Most of the barriers discussed in this study do not concern food safety knowledge. Thus,

168 providing food safety training that focuses only on improving knowledge may not assist

169 employees in overcoming barriers. Other studies indicated that other factors needed to be

170 investigated in training programs other than increasing knowledge alone $(7,15)$.

171 Managers should ensure that employees receive food safety training on a regular basis.

172 Training should not only focus on increasing knowledge. The results of this research shows that

173 employees list having a poor attitude regarding food safety as a barrier to proper food handling;

174 therefore educating employees about the consequences of improper food handling might improve

175 attitudes toward food safety in general. By realizing the consequences, employees may be less

176 likely to perceive food safety practices as an inconvenience. Signs could be placed in food

177 production areas with persuasive messages about the consequences of not implementing food

178 safety practices. Participants in another study reported that signs in handwashing areas and

179 restrooms were important reminders to employees (15). Managers should also monitor

180 employees' food safety behaviors and encourage all employees to practice proper food safety

181 practices by giving verbal reminders often, being positive role models, and reinforcing 
182 employees’ food safety behaviors with verbal praise. It is also important for managers to instruct

183 their employees on proper food safety techniques when they observe employees engage in

184 negative food safety behaviors during their daily activities.

185 Other researchers have found time pressures to be a factor that influences food safety

186 practices such as washing hands, changing gloves, cleaning cutting boards, checking

187 temperatures, and cooling/reheating foods (9). Training sessions should focus on educating

188 employees that properly performing the practices does not take as much time as perceived (e.g.,

189 it only takes 4 minutes for an employee to wash their hands 12 times an hour or it only takes 30

190 seconds to take the end-point cooking temperature of a food item). Foodservice managers, RDs,

191 and DTRs should incorporate food safety practices into employees’ daily routines to eliminate

192 the perceptions that time constraints are a barrier to performing proper food safety practices.

Other researchers have also found that restaurant employees reported that inadequate

194 supplies and problems with access to sinks were barriers to handwashing (15). Managers, RDs,

195 and DTRs should ensure that adequate supplies are kept in inventory so that lack of adequate

196 resources is not a barrier. Inventory should be closely monitored to assure that hand soap, paper

197 towels, thermometers, and cleaning and sanitizing supplies are available so employees can

198 follow food safety guidelines. Participants in another study suggested that having managers

199 more involved in food safety training and making sinks more accessible were factors that

200 promoted handwashing (15).

201 Results were used to develop and implement interventions for foodservice establishments

202 to overcome perceived barriers that training does not appear to address. Intervention materials

203 included food safety posters that contained "how to" and persuasive "did you know” messages. 
206 Results can only be generalized to the restaurants in the three states participating in this

207 study. This study only focused on restaurant employees with food production responsibilities. It

208 did not involve other employees such as servers or maintenance staff. It would be interesting to

209 determine barriers perceived by other employees given that these employees also affect the

210 safety of food. It also would be important to investigate barriers perceived by employees who

211 work in other foodservice segments such as healthcare, schools, universities, childcare, and

212 senior living communities. Future research could be conducted with RDs, DTRs, and

213 foodservice managers to assess their perceptions of barriers to following proper food safety

214 practices. The differences in managers' and employees’ perceptions of barriers could be

215 determined and specific interventions identified. 


\section{References}

217 1. National Restaurant Association. Restaurant Industry Facts. Available at: http://www.restaurant.org/research/ind_glance.cfm. Accessed May 14, 2007.

2. 2007 restaurant industry forecast. Washington, DC: National Restaurant Institute; 2006;1931400-62-8.

221 3. Centers for Disease Control and Prevention. Preliminary FoodNet data on the incidence of Accessed November 29, 2006.

4. Harrington, RE. The role of employees in the spread of foodborne disease: Food industry infection with pathogens transmitted commonly through food - 10 states, United States, 2005. MMWR Morb Mortal Wkly Rep. 2006; 55(14). Available at: http://www.cdc.gov/mmwr/preview/mmwrhtml/mm5514a2.htm?s_cid=mm5514a2_e.

5. Food and Drug Administration. FDA report on the occurrence of foodborne illness risk factors in selected institutional foodservice, restaurant, and retail food store facility types. 2004. Available at: http://www.cfsan.fda.gov/ acrobat/retrsk2.pdf. Accessed June 25, 2006.

6. Lynch RA, Elledge BL, Griffith CC, Boatright DJ. A comparison of food safety knowledge among restaurant managers, by source of training and experience, in Oklahoma County, Oklahoma. Journal of Environmental Health. 2003; 66(2): 9-14.

7. Cohen E, Reichel A, Schwartz Z. The efficacy of an in-house training program: Statistical measurement and practical conclusions. Journal of Hospitality \& Tourism Research. 2001; 25(1): 5-16. 
8. Cochran-Yantis D, Belo P, Giampaoli J, McProud L, Everly V, Gans J.

Attitudes and knowledge of food safety among Santa Clara County, California restaurant operators. Journal of Foodservice Systems. 1996; 9: 117-128.

9. Green LR, Selman C. Factors impacting food workers’ and managers’ safe food preparation practices: A qualitative study. Food Protection Trends. 2005; 25: 981-990.

10. Pilling VK, Brannon LA, Roberts KR, Shanklin CW, Howells AD. Using the Theory of

11. Brannon, LA, Pilling, VK, Roberts, KR, Shanklin, CW, Howells, AD. Appreciation of food safety practices based on level of experience. Journal of Foodservice Business Research. in press.

12. Pilling, VK, Brannon, LA, Shanklin, CW, Howells, AD, Roberts, KR. Identifying specific beliefs to target to improve restaurant employees’ intentions for performing three important food safety behaviors. J Am Diet Assoc. in press.

13. Ajzen I. The theory of planned behavior. Organizational Behavior and Human Decision Processes. 1991;50:179-211.

14. Francis JJ, Eccles MP, Johnston M, Walker A, Grimshaw J, Foy R, Kaner EFS, Smith L, Bonetti D. Constructing questionnaires based on the theory of planned behaviour: A manual for health services researchers. Newcastle upon Tyne, United Kingdom: Centre for Health Services Research; 2004;0-9540161-5-7. 
260 15. Pragle AS, Harding AK, Mack JC. Food workers' perspectives on handwashing behaviors and barriers in the restaurant environment. Journal of Environmental Health. 2007;

262 69(10): 27-32. 
Table 1-Barriers Identified by Focus Group Participants for Three Food Safety Practices

\begin{tabular}{|c|c|c|c|c|c|c|}
\hline \multirow{2}{*}{ Barrier } & \multicolumn{2}{|c|}{$\begin{array}{c}\text { Cleaning and } \\
\text { Sanitizing } \\
\end{array}$} & \multicolumn{2}{|c|}{ Handwashing } & \multicolumn{2}{|c|}{ Using a Thermometer } \\
\hline & \multicolumn{6}{|c|}{ Number of Focus Groups Identifying Each Barrier } \\
\hline 1. Time Constraints & 10 & 18 & 10 & 14 & 9 & 7 \\
\hline 2. Inadequate Training/Knowledge & 8 & 10 & 1 & 8 & 6 & 13 \\
\hline A. not knowing consequences of not doing it & 6 & -- & -- & -- & -- & -- \\
\hline C. not understanding the necessity of it & -- & -- & 3 & -- & -- & -- \\
\hline D. not knowing temperatures & -- & -- & -- & -- & 7 & 3 \\
\hline E. not knowing how to calibrate thermometers & -- & -- & -- & -- & 5 & -- \\
\hline 3. Forgetting/Having to Remember & 2 & 6 & 4 & 2 & 1 & -- \\
\hline A. no signs/no reminders & -- & -- & 3 & -- & -- & -- \\
\hline 4. Lack of Adequate Resources & 6 & 4 & 4 & 13 & -- & -- \\
\hline E. lack of sanitizer & -- & 1 & -- & -- & -- & -- \\
\hline F. lack of enough sinks & -- & -- & 5 & -- & -- & -- \\
\hline G. lack of soap and paper towels & -- & -- & 4 & -- & -- & -- \\
\hline H. lack of working thermometers & -- & -- & -- & -- & 9 & 11 \\
\hline I. not enough thermometers & -- & -- & -- & -- & -- & 10 \\
\hline J. no cleaning swabs for thermometers & -- & -- & -- & -- & -- & 2 \\
\hline 5. Management and Employees Don’t Care & 8 & 13 & -- & -- & 1 & 5 \\
\hline A. other employees criticizing you & -- & 3 & -- & -- & -- & -- \\
\hline B. being told it is not cost effective & -- & 2 & -- & -- & -- & 2 \\
\hline C. managers not monitoring & -- & 5 & 3 & 1 & 4 & 3 \\
\hline
\end{tabular}


D. managers/other employees being bad examples

E. not being held accountable

6. Competing Tasks

A. impatient guests

B. impatient managers

7. Inconvenient/Hassle/Easier Not to Do

8. No incentive/No Desire to Do It

9. No habit

10. Other

A. creates more work

B. language barriers

C. resources in inconvenient locations

D. dry skin

E. complicated/hard to read thermometers

\begin{tabular}{|c|c|c|c|c|c|}
\hline -- & 4 & -- & 3 & -- & -- \\
\hline -- & 2 & -- & -- & -- & -- \\
\hline 3 & 7 & 6 & 7 & 2 & -- \\
\hline 1 & -- & -- & -- & 1 & -- \\
\hline 2 & 6 & -- & 3 & 1 & -- \\
\hline 4 & 4 & 1 & 3 & 3 & 2 \\
\hline -- & 15 & -- & -- & -- & -- \\
\hline 1 & -- & -- & -- & -- & -- \\
\hline -- & 4 & -- & -- & -- & -- \\
\hline -- & 1 & -- & -- & -- & 1 \\
\hline -- & -- & 8 & 8 & 5 & 2 \\
\hline -- & -- & 7 & 11 & -- & -- \\
\hline -- & -- & -- & -- & -- & 1 \\
\hline
\end{tabular}

${ }^{\mathrm{a}}$ Group A: A series of 10 focus groups with 34 employees whose job involved food production from local restaurants.

${ }^{\mathbf{b}}$ Group B: A series of 20 focus groups with 125 employees whose job involved food production from restaurants within a 300-mile radius of the research university. 